\title{
Small Celestial Body Image Restoration Based on Spectrum Characteristics
}

\author{
TAO Tianyuan ${ }^{1, a}$, *KANG Zhiwei ${ }^{1, b}$, LIU Jin ${ }^{2, c}$ \\ ${ }^{1}$ College of Computer Science and Electronic Engineering, Hunan University \\ ${ }^{2}$ College of Information Science and Engineering, Wuhan University of Science and Technology \\ ${ }^{a}$ E-mail: tytao@hnu.edu.cn b ${ }^{\text {E E-mail: jt_zwkang@hnu.edu.cn }{ }^{\mathrm{c}} E-m a i l: ~ l i u j i n @ w u s t . e d u . c n ~}$
}

Keywords: spectral characteristics; image restoration; NSCT; minimum amplitude distance.

\begin{abstract}
To remove the blur of the image from optical navigation camera, a image restoration method based on spectrum characteristics is proposed. In this method, image denoising pretrea tment is first implemented by the NSCT filter. Then, to obtain the minimum amplitude distanc e, the paper optimizes the image spectrum characteristic by using the simulated annealing al gorithm. And then the minimum amplitude distance can be obtained. There the point spread fu nction is established. Finally, blurred image is recovered by the Lucy - Richardson algorithm.T he recovery quality is evaluated by the Laplace gradient and the average gray level gradient. $\mathrm{T}$ he simulation results demonstrate that the proposed method in the paper improves the quality of the restored small celestial body image, and the visual effect is also very ideal.
\end{abstract}

\section{1、 Introduction}

In the process of the deep space exploration, the imaging system of the optical optical camera is vulnerable to the flight speed and the cosmic rays [1], which cause the image fuzzy and inconvenience for image information analysis. Thus, how to restore fuzzy image is a difficulty of image information process in the deep space exploration.

The image restoration of small celestial body mainly consists of the image denoising pretreatment and the image degradation process estimation. At present, there are many relevant researches about the image denoising pretreatment. For instance, the median filtering algorithm has a very good effect on the salt and pepper noise, but it is not ideal to other interference noises [1]. The bilateral filter algorithm can eliminate the image noise, while it cannot make image detail intact [2]. The degradation function is important to determine the degradation process. The degradation function of the optical camera is the point-spread function (PSF) [3]. For the point- spread function, a lot of researches have been done by domestic and foreign scholars. X. H. Wang et al, considered that the distance between the spectrum center and the adjacent black belt is inversely proportional to the blurred length [4]. But it is only a project of the degradation function estimation, and does not have a specific quantitative formula. N. Norouzi et al., put forward a method of derivative, which can detect image blurred length. However, the error is very serious under the condition of noise [5].

For the image blur problem caused by the optical camera, this paper proposes the NSC algorithm, which mainly includes image denoising pretreatment based on the NSCT filter and fuzzy regression model construction based on the spectrum characteristics.

\section{2、NSC algorithm}

\section{1、NSCT filter}

The non - subsampled contourlet (NSCT) filter is composed of the non - subsampled pyramid (NSP) [6] and the non - subsampled direction filter group (NSDFB) [7]. The redundant representation is adopted by the NSCT filter, which can effectively capture image characteristics, and distinguish between noise and image edge [9]. Thus, it has a very good denoising effect.

The non-subsampled pyramid (NSP) is used for multiscale pyramid decomposition without 
up-sampling and down-sampling, which reduces distortion induced by sampling, and presents translation invariance. The non - subsampled direction filter group (NSDFB) is related to direction analysis. By the direction analysis, we can obtain the NSCT coefficient composed by the singularity in the same direction. Finally, the NSCT coefficient is utilized for inverse transform. Consequently, we realize image de-noising.

\section{2、 Spectrum characteristics-based fuzzy regression model construction}

The degradation model of the blurred image can be expressed as follow :

$$
g(x, y)=f(x, y) \cdot h(x, y)+n(x, y)
$$

where $g(x, y)$ is the blurred image. $f(x, y)$ is the original image. $h(x, y)$ is the point-spread function. $n(x, y)$ is the noise.

In order to observe small celestial body image intuitively, the frequency domain expression of equation (1) can be expressed as follow:

$$
G(u, v)=F(u, v) \cdot H(u, v)+N(u, v)
$$

In the area of the deep space exploration, the prior knowledge of the small celestial body image is not enough. Thus, the spectrum characteristics of the image are analyzed for the point-spread function. In this paper, we adopt the point-spread function to build the degradation model. The point spread function (PSF) is determined by the fuzzy length and the fuzzy angle.

We can adopt a binaryzation method to process the spectrum map. As a result, the gradient $\theta$ of the bright spot can be observed. We take $\theta$ as the fuzzy angle of the image [4].

Next, we analyze the fuzzy length of the image. This paper takes the real part of the image as research subject. The real part Fourier transform of the image is as follow:

$$
F(\omega)=\int_{-\infty}^{+\infty} A e^{-j \omega t} d t=2 \pi A \delta(\omega)
$$

where $A$ means the real part of the image. According to equation (1). The real spectrum map is axial symmetric figure, and the symmetric center is a impulse function. If the image appears blur, the corresponding amplitude will deviate. We take the minimum amplitude distance as the fuzzy length. The specific identification steps are as follows:

1) After Fourier transform and Fourier inverse transform, we can get real part spectrum map.

2) The simulated annealing algorithm is a kind of intelligent data optimization algorithm [9], which is used to filter the real part spectrum data. Two minimum amplitudes $F_{1} \quad F_{2}$ are obtained.

3) According to formula (1), the corresponding frequencies with respect to two minimum amplitudes $F_{1} F_{2}$ are $\omega_{1}$ and $\omega_{2}$, and $\omega_{1}<\omega_{2}$. Thus, the fuzzy length $d=\omega_{2}-\omega_{1}$

The fuzzy length $d$ and the fuzzy angle $\theta$ are known. Discretization matrix of the point-spread function can be expressed as:

$$
\begin{aligned}
& \mathrm{y}=\left[\frac{m}{n} \cdot x\right], x=0,1, \cdots, n-1 \\
& y=\left[\frac{n}{m} \cdot x\right], x=0,1, \mathrm{~L}, n-1
\end{aligned}
$$

where $m$ and $n$ are the fuzzy measurement of horizontal direction and vertical direction, respectively. They expressions are as follows:

$$
\begin{aligned}
& m=d \cdot \cos \theta \\
& n=d \cdot \sin \theta
\end{aligned}
$$

when $n>m$, formula (4) is functional. When $n<m$, formula (5) is functional. [ ] is integer-valued notation.

\section{3、 Implementation steps of the NSC algorithm}

In summary, the flow chart of the NSC algorithm is shown as Fig.1 


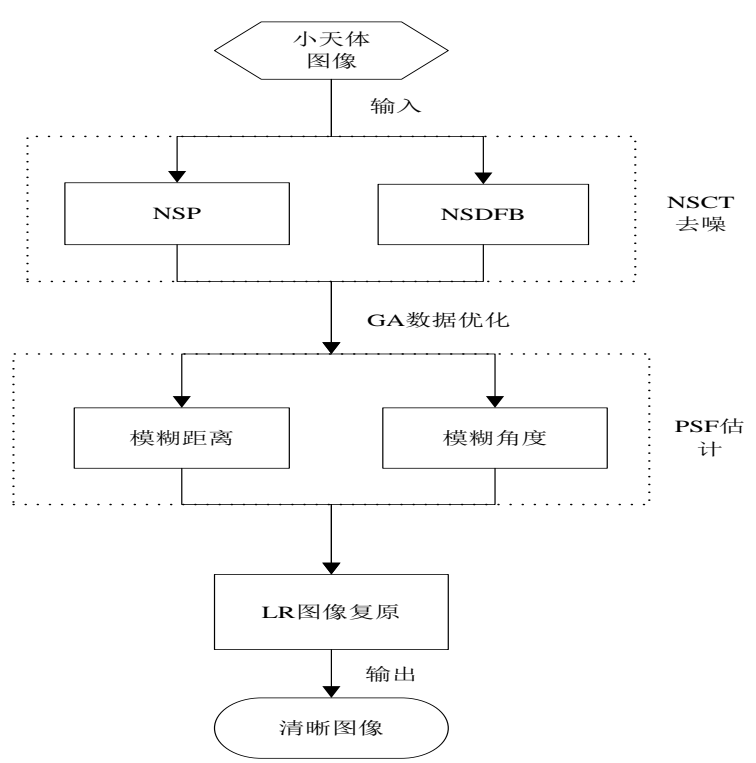

Fig.1 Flow chart of the algorithm

The NSC algorithm includes the NSCT denoising, the PSF estimation and the LR restoration. The specific implementations are as follows:

1) NSCT denoising: According to section A, the Non - subsampled pyramid (NSP) is used to decompose the small celestial body image. The Non - subsampled direction filter group (NSDFB) is used to decompose the high frequency sub-band. So we can get the NSCT coefficient. Finally, the coefficient is used for the NSCT inverse transform.

2) PSF estimation: According to section $B$, the gradient of the bright spot is estimated, and the angle is $\theta$. We take the spectrum characteristic $\theta$ as the fuzzy angle of the image. Then the simulated annealing algorithm is used to optimize the real part spectrum data of the fuzzy image. Two minimum amplitudes are obtained. We estimate the fuzzy length on the basis of the distance between two minimum amplitudes. At the last, the point-spread function is determined by the fuzzy length and the fuzzy angle. Namely, the fuzzy regression model is established.

3) LR restoration: After the fuzzy regression model is built, the LR algorithm is used for image restoration.

\section{4、 Simulation results}

The simulation experiment data devices from National Aeronautics and Space Administration (NASA)[10]. We adopt two orbits: (a) The orbital altitude is 38 kilometers, which was taken on January 11, 2001. (b) The orbital altitude is 124 miles, which was taken on November 3, 2000.



(a) Orbit with $38 \mathrm{~km}$



(b) Orbit with 124 miles

Fig 2. Simulation image

In order to demonstrate the effectiveness and stability of the NSC algorithm, we compare it with the CA method. For the CA algorithm, the bilateral filter is used for image denoising. The method of derivative is used to estimate the point-spread function according to the literature [6]. For the 
NSC method, we utilize the NSCT filter for image denoising. The spectral characteristics are used to estimate the point-spread function. The simulation results are shown in figure 3. From figure.3, we can see that figure (b) shows more detailed characteristics and has better visual effect.
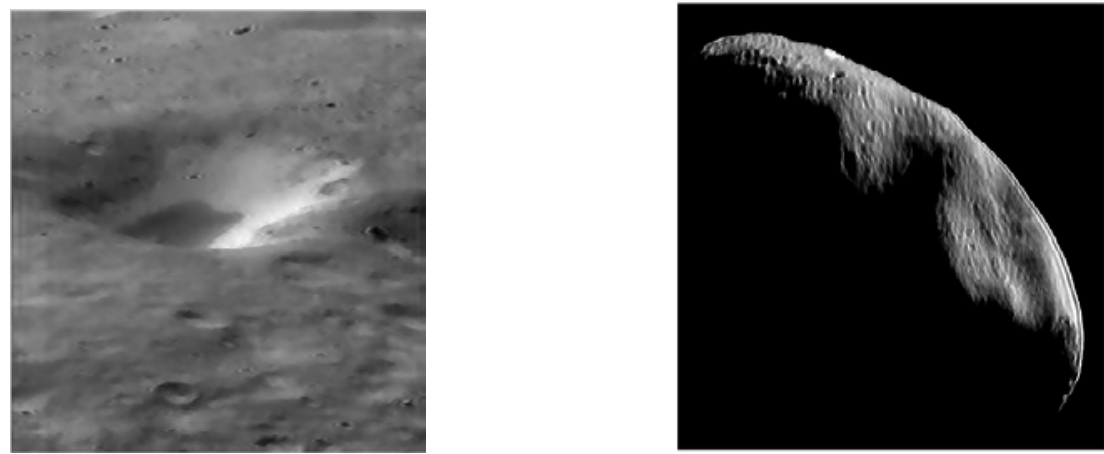

(a). Restoration image by CA algorithm
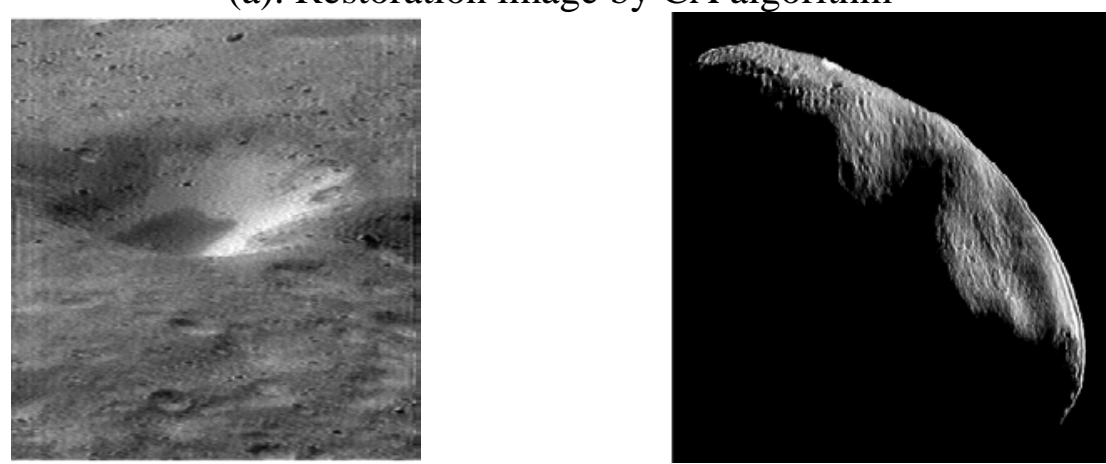

(b). Restoration image by NSC algorithm

Fig.3 Comparison of restoration image

For objective evaluation of the restoration performance of the proposed method, we use Laplace gradient (LS) to evaluate the quality of recovery, which is defined as follow:

$$
L S=\frac{\sum_{i=2}^{M-1} \sum_{j-2}^{N-1} \mid 8 g(i, j)-g(i-1, j-1)-g(i-1, j)-g((i-1, j+1)-\cdots-g(i+1, j+1) \mid}{(M-2)(N-2)}
$$

The accuracy of restoration is also evaluated by average gray level gradient (GMG), the function is as follow:

$$
G M G=\sum_{i=1}^{M-1} \sum_{j=1}^{N-1} \sqrt{\frac{[g(i, j+1)-g(i, j)]^{2}+[g(i+1, j)-g(i, j)]^{2}}{2}}
$$

where $g(i, j)$ is the restoration image. $M \times N$ is the size of the image. LS and GMG are applicable without detailed image information, and high-value represents that the restoration effect is ideal. The results of simulation are shown in Tab.1, including five groups Eros images of different altitudes. From Tab.1, it can be seen that LS and GMG of the NSC algorithm are higher than 3 orders of magnitude those of adopted by the CA algorithm. The result suggests that the NSC method outperforms other method with obvious LS and GMG difference.

Tab. 1 Comparison of recovery quality

\begin{tabular}{|c|c|c|c|c|}
\hline & \multicolumn{2}{|c|}{ LS } & \multicolumn{2}{c|}{ GMG } \\
\hline Algorithm & CA & NSC & CA & NSC \\
\hline a & 3987.7 & 7282500 & 0.000064597 & 0.0023575 \\
\hline b & 44504 & 24557000 & 0.0000036804 & 0.00028747 \\
\hline c & 3254.2 & 5086600 & 0.000032392 & 0.0030966 \\
\hline d & 845.25 & 527130 & 0.000027625 & 0.0082610 \\
\hline e & 7547.8 & 5648700 & 0.000076743 & 0.0028659 \\
\hline
\end{tabular}




\section{5、Conclusion}

In this paper, a spectrum characteristics-based image restoration method is proposed, which utilizes the NSCT filter as the noise filter, and bases on the spectrum characteristics to built fuzzy regression model. The simulation results demonstrate that this method is an efficient image restoration method, and it has a high accuracy with small celestial body image.

\section{References}

[1] S. Bhaskaran, N. Masrtodemos, J. E. Riedel. et al, Optical Navigation for the Stardust Wild 2 Encounter, Proceedings of the $18^{\text {th }}$ International Symposium on Space Flight Dynamics. ( 2004) 455-460.

[2] W. Shao. Image-Based Small Body Parameters Estimation and Spacecraft Autonomous Navigation , Harbin Institute of Technology. (2009).

[3] M. E. Moghaddam, M. Jamzad, Finding point spread function of motion blur using radon transform and modeling the motion length, Signal Processing and Information Technology. (2005) 862-866.

[4] X. H. Wang, R. C. Zhao, Estimation the PSF of motion -blurred images, Computer Applications. (2001) , 09: 40 -41.

[5] N. Norouzi, M. E. Moghaddam, Motion blur identification using image derivative, IEEE International Symposium on Signal Processing and Information Technology. (2008) 380-384.

[6] X. M. Zhang , J. S. Song , Z. X. Yi , et al, Extraction and Recognition of Bridge over Water in High Resolution SAR Image, Advances in Multimedia,Software Engineering and Computing. Springer Berlin Heidelberg. ( 2012) 19-24.

[7] M. LI, T. WANG, The nonsubsampled complex contourlet transform for image denoising, Computer Engineering and Technology (ICCET). (2010) 267-270.

[8] Y. Q. Wu, J. P. Shi, F. X. Tao, A NSCT-Domain Watermarking Algorithm Against Geometric Attacks Based on SIFT and NMF-SVD, Journal of Electronic Measurement and Instrument. 29(7) (2015) 961 -969.

[9] S. Lyden, M. E. Haque, A Simulated Annealing Global Maximum Power Point Tracking Approach for PV Modules Under Partial Shading Conditions. IEEE Transactions on Power Electronics. (2016) 4171-4181.

[10] http://near.jhuapl.edu/iod/archive.html 